rheuma plus $2019 \cdot 18: 79$

https://doi.org/10.1007/s12688-019-0265-8

(c) Springer-Verlag GmbH Austria, ein Teil von Springer Nature 2019

\section{Burkhard Leeb}

Institut für Klinische Rheumatologie, Karl Landsteiner Gesellschaft, Hollabrunn, Österreich

\title{
Adhärenz - für Patienten und Ärzte dasselbe?
}

\section{Liebe Leserinnen und Leser!}

Alle wollen für die Patienten das Beste: Ärzte, Sozialversicherung, Politik, Pharmaindustrie, Selbsthilfegruppen usw. Manchmal hat es fast den Anschein, unser System funktionierte ohnehin hervorragend - wenn, ja wenn, nicht die Patienten wären! Denn ihre Wünsche und Gedanken sind individuell sehr verschieden, abhängig von der Erkrankung, dem sozioökonomischen Background, dem Bildungsgrad usw. Eines aber ist sicher: alle Patienten, übrigens auch Ärzte in dieser Position, wollen für sich das Beste und - so paradox das klingen mag - sie nehmen, generell gesehen, ihre Medikamente nicht entsprechend der Verordnung ein.

Den ketzerischen Gedanken, dass viele Patienten mit diesem Verhalten Recht haben könnten, darf man ja nicht einmal im Ansatz haben, denn dann wäre ja die Adhärenz (als Maß für die Erfüllung der therapeutischen Vorgaben) schlecht. Das geht von der meiner Meinung nach etwas naiven Vorstellung aus, dass in klinischen Studien die Medikation korrekt eingenommen würde, obwohl man aus Hypertonie-Studien das Gegenteil wissen sollte. Und so hielt die Adhärenz auch in der Rheumatologie Einzug - mit dem Unterschied, dass wir schmerzhafte Erkrankungen behandeln; und so viele Masochisten unter den Patienten wird es schon nicht geben.

Etwas anders allerdings verhält es sich mit der Harnsäuresenkung bei Gicht-Patienten. Wenn der Gichtschub lange genug vorbei ist, verblasst die Erinnerung an den Schmerz und die Disziplin in diätetischer Hinsicht und auch die Einnahme der Medikamente lässt nach. Aufklärung tut not, aber Patienten vertrauen anderen Health Care Providern mehr als Ärzten (warum wohl?). Mit zwar redlich gemeinten, aber sehr praxisfernen Empfehlungen für Gruppen von Betroffenen, wird der oben beschriebene Umstand wohl nicht zu beheben sein. Verständliches Eingehen auf den einzelnen Patienten mit seinen Fragen, Wünschen, Ängsten wäre der wahrscheinlich erfolgversprechende Weg; individualisierte Behandlung und auch Interpretation der Adhärenz tut not.

Ein Bericht vom Rheuma-Update 2019 in Wiesbaden beschäftigt sich mit der Anwendung von Antidepressiva bei rheumatologischen Patienten. Darüber hinaus wird auch über Therapiereduktion bei optimalem Therapieverlauf entzündlich rheumatischer Erkrankungen berichtet, diesmal aus sonographischer Sicht. Infektionen unter Biologikatherapie werden vor allem zu Beginn der Behandlung häufiger festgestellt, die Rolle von Impfungen, insbesondere im Licht der neuen Zoster-Impfung mit Totimpfstoff wurde in Wiesbaden besonders betont, ebenso wie die Rolle der oralen Mikrobiota bei entzündlich rheumatischen Erkrankungen.

Chronische Entzündung ist generell nicht gesund. Dies gilt auch für die jüngsten Patienten, so dass dem Beitrag von Jürgen Brunner, Innsbruck über den Morbus Still, auch im Sinne der Awareness entsprechende Beachtung geschenkt werden sollte. Eine interessante Fallbeschreibung aus dem Gebiet der Vaskulitiden - das Auge betreffend wird unter der Rubrik „Aus der Praxis“ Bestandteil dieser Ausgabe sein.

Wir hoffen, auch mit dieser Ausgabe wieder Ihr Interesse wecken zu können, um dem Ziel von rheuma plus nahe zu kommen, eine fruchtbringende, offene und redliche Diskussion in Gang zu bringen, aus der alle Nutzen ziehen können. Wie immer an dieser Stelle möchten wir Sie herzlich dazu einladen, uns Ihre Meinung zu den Beiträgen dieser Ausgabe, wie auch $\mathrm{zu}$ allen rheumatologischen Themen, die Sie für relevant oder für diskussionswert halten, zu schicken. Sie können in jedem Fall auf einen fairen Review mit entsprechendem Feedback vertrauen. Für Kritik, Hinweise, Zustimmung wie auch für jeden anderen Beitrag bedanke ich mich schon im Voraus.

Herzlichst, Ihr Burkhard Leeb

\section{Korrespondenzadresse

Burkhard Leeb
Institut für Klinische
Rheumatologie, Karl
Landsteiner Gesellschaft
Babogasse 20, 2020 Holla-
brunn, Österreich
leeb.rheuma@aon.at

Interessenkonflikt. B. Leeb gibt an, dass kein Interessenkonflikt besteht.

Hinweis des Verlags. Der Verlag bleibt in Hinblick auf geografische Zuordnungen und Gebietsbezeichnungen in veröffentlichten Karten und Institutsadressen neutral. 